\title{
Desempenho e metabolizabilidade de dietas em codornas alimentadas com níveis crescentes de óleo ácido de soja
}

\author{
[Performance and metabolizability of diets in quails fed with increasing levels of soybean acid oil] \\ A.A.P. Roll ${ }^{1}$, J. Forgiarini ${ }^{1}$, C. Bavaresco ${ }^{1}$, V.F.B. Roll ${ }^{2}$, N.J.L. Dionello ${ }^{2}$, F. Rutz ${ }^{2}$ \\ ${ }^{1}$ Universidade Federal de Pelotas - Pelotas, RS \\ ${ }^{2}$ Programa de pós-graduação - Universidade Federal de Pelotas - Pelotas, RS
}

\begin{abstract}
RESUMO
Objetivou-se avaliar os efeitos do óleo ácido de soja (OAS) sobre coeficientes de extinção específica da oxidação (K232 e K270) e a metabolizabilidade das dietas e o desempenho produtivo em codornas. Um total de 80 codornas de duplo propósito foram usadas num experimento com duração de 56 dias. Foi utilizado um delineamento completamente ao acaso com cinco níveis de tratamentos para OAS $(0,25,50$, 75 e $100 \%$ ), com oito repetições de duas aves cada, como substituto ao óleo de soja refinado (OS) incluído até um nível máximo de $8 \%$ na formulação da dieta. O índice de acidez e os coeficientes K232 e K270 mostraram um efeito linear crescente $(\mathrm{P}<0,001)$ com o aumento dos níveis de inclusão de OAS na formulação das dietas. Os coeficientes K232 e K270 não foram alterados com o armazenamento das rações durante 7 dias. A suplementação com OAS durante 56 dias não afetou o desempenho produtivo das codornas. Os resultados não mostraram efeitos do OAS sobre a metabolizabilidade de ingredientes, exceto para gordura bruta que exibiu um efeito quadrático decrescente até o nível de $50 \%$ de substituição do óleo de soja por OAS $\left(y=94,31-1,186 x+0,018 x^{2}, R^{2}=0,28, P=0,02\right)$. Em conclusão, a inclusão de até $8 \%$ de OAS na dieta durante um período curto de tempo (56 dias) não produz efeitos adversos no desempenho produtivo de codornas.
\end{abstract}

Palavras chave: codornas, desempenho, metabolizabilidade, óleo ácido de soja

\begin{abstract}
This study evaluated the effects of soybean acid oil (OAS) on oxidation products specific extinctions coefficients (K232 and K270) and metabolizability of diets and productive performance in quails. A total of 80 dual-purpose quails were used in a 56-days experiment. A completely randomised design with five treatment levels of OAS (0, 25, 50, 75 and 100\%) as a dietary replacer for soybean oil (OS) included up to $8 \%$ in the diet formulation were used, with eight replicates of two birds each. The acidity index and $K 232$ and $K 270$ coefficients showed a crescent linear effect $(P<0.001)$, with increasing levels of OAS in the diet formulation. There were no changes in K232 and K270 values during 7-days of diet storage. Supplementation with OAS during 56 days has no effect on quail's productive performance. The results showed no effects of OAS on metabolizability of ingredients except for crude fat that exhibited a quadratic effect that was decrescent to the level of $50 \%$ of OAS supplementation as soybean oil replacer $\left(y=94.31-1.186 x+0.018 x^{2}, R^{2}=0.28, P=0.02\right)$. In conclusion, dietary inclusion of OAS up to $8 \%$ in the diet during a short period of time (56-days) produce no adverse effects on productive performance of quails.
\end{abstract}

Keywords: quails, performance, metabolization, soybean acid oil

Recebido em 3 de março de 2017

Aceito em 3 de abril de 2017

E-mail: apiroll@yahoo.es 


\section{INTRODUÇÃO}

O aporte de óleos vegetais nas formulações de ração tem sido uma ferramenta muito utilizada pelos nutricionistas, pois além de promover a redução da pulverulência aumenta a densidade nutricional das rações possibilitando uma melhora no desempenho e na conversão alimentar das aves (Lara et al., 2005). Neste sentido, a busca por gorduras de qualidade a preços competitivos tem sido um dos principais objetivos dos nutricionistas na produção animal.

Fontes alternativas de gordura, tais como os óleos ácidos, tem despertado interesse por sua possível utilização como fontes energéticas na produção de ração animal. Os óleos ácidos são co-produtos derivados da refinamento de óleos podendo apresentar de $75 \%$ a $95 \%$ de ácidos graxos presentes nos óleos de que se originam (Freitas et al., 2005). Estes óleos são caracterizados por apresentarem grande quantidade dos ácidos graxos na forma de ácidos graxos livres (AGL: 40-90\%) (Vieira et al, 2002; Freitas et al., 2005) e são conhecidos pela sua menor digestibilidade devido à falta de monoglicerídeos (MG) suficientes para promover a absorção (Raber et al., 2009). Por outro lado, também é um resíduo da indústria que precisa ser reciclado com o objetivo de reduzir a carga sobre o meio ambiente (Vieira et al., 2002).

Embora a utilização da energia a partir de dietas ricas em triglicerídeos (TG) seja considerada superior em comparação com as dietas ricas em AGL, alguns estudos tem reportado que os AGL não são os responsáveis por essa ineficiência. De Groote (1968) trabalhando com dietas ricas em AGL, que foram adequadamente estabilizados por antioxidantes, não encontraram nenhum efeito negativo sobre $o$ desempenho de pintinhos, independentemente do conteúdo de AGL. Da mesma forma, Lewis e Payne (1963) e Waldroup et al. (1995) trabalhando com dietas suplementadas com diferentes níveis de AGL, não encontraram diferenças no desempenho de frangos de corte. Treat et al. (1960) em estudo alimentando galinhas poedeiras com dietas com níveis elevados em AGL, não observaram efeitos adversos sobre a produção de ovos.
Mais recentemente, Vieira et al. (2002) estudando a inclusão de óleo ácido de soja (OAS) em substituição total ou parcial ao óleo de soja, não encontraram diferenças no desempenho de frangos que receberam $8 \%$ de inclusão de óleo como fonte de energia na dieta. Os mesmos autores relatam que a deficiência de resultados de energia metabolizável confiáveis desses óleos, pode ser uma provável causa do reduzido uso do OAS nas rações das aves visando redução de custos com a formulação.

Sabendo-se que a velocidade de passagem do alimento pelo trato gastrointestinal das codornas é mais rápido com relação a outras aves, isto poderia afetar a digestibilidade dos nutrientes da dieta (Sakamoto et al., 2006), consequentemente aumentando a deficiência no aproveitamento e utilização de alguns nutrientes e reduzindo assim o desempenho produtivo dos animais. Segundo Sakamoto et al. (2006), a digestibilidade de nutrientes e o seu valor energético são influenciados pelo rápido tempo de passagem da digesta pelo intestino das codornas, de 1 a 1,5 horas, contra 3 a 5 horas em galinhas, sendo que as codornas são capazes de aproveitar melhor a energia provinda da fibra da ração devido ao maior tamanho relativo do ceco.

Portanto, como os alimentos alternativos tem sido estudados na sua maioria em rações para frangos de corte e galinhas de postura (Murakami e Furlan, 2002), aliado a inconsistência na composição dos ácidos graxos desses ingredientes e a incerteza quanto aos seus valores energéticos e econômicos, a falta de literatura sobre a utilização do OAS na dieta de codornas expõe uma lacuna dentro da nutrição dessas aves. Sendo assim, o objetivo do presente trabalho é caracterizar o OAS e investigar o seu potencial de utilização na nutrição de codornas através do desempenho produtivo e da metabolizabilidade das dietas.

\section{MATERIAL E MÉTODOS}

O experimento foi conduzido no Laboratório de Ensino e Experimentação Zootécnica Professor Renato Rodrigues Peixoto, do Departamento de Zootecnia/FAEM/ UFPEL. Este trabalho foi realizado de acordo com as normas éticas, sendo aprovado (processo $\mathrm{n}^{\circ}$ 23110.001433/2015-25) pela Comissão de Ética em Experimentação 
Animal (CEEA)/Universidade Federal de Pelotas-UFPel sob o n ${ }^{\circ}$ CEEA 1433-2015.

Foram utilizadas 80 codornas Coturnix coturnix coturnix. Antes de dar início ao experimento todas as codornas foram pesadas individualmente e distribuídas ao acaso nas gaiolas experimentais. As codornas foram pesadas no primeiro e no último dia de cada ciclo experimental (correspondente a um período de 28 dias). Para a pesagem das aves foi utilizada uma balança analítica de precisão $(0,01 \mathrm{~g})$ e utilizando-se de um balde com tampa (polietileno/1L) para facilitar o manejo durante a pesagem.

As aves utilizadas neste experimento eram oriundas da $17^{\mathrm{a}}$ geração de um programa de melhoramento por peso corporal de uma linhagem de codornas (Coturnix coturnix coturnix) que são selecionadas pelo peso corporal aos 21 dias de idade (Dionello et al., 2008).

As aves foram alojadas em gaiolas de arame galvanizado em uma sala climatizada com temperatura controlada de $23^{\circ} \mathrm{C} \pm 1$. As gaiolas eram distribuídas em seis andares, suspensas e dispostas em linhas sobrepostas, contendo comedouros individuais do tipo calha, colocado externa e longitudinalmente na frente da gaiola, além de bebedouro do tipo nipple localizado no fundo de cada gaiola.

A iluminação da sala foi feita com lâmpadas fluorescentes e controlada por relógio timer para fornecer 17 horas de luz diárias. A climatização foi feita através de aparelho de ar condicionado do tipo spliter e o sistema de ventilação feito através de janelas reguláveis. As temperaturas e umidades, do interior da sala foram obtidas através de um termohigrômetro digital localizado no centro da bateria de gaiolas. Os registros foram realizados pela manhã em torno de $8 \mathrm{~h}$, ou seja, antes da coleta dos ovos e arraçoamento das aves.

As dietas experimentais foram formuladas à base de milho e farelo de soja com a composição nutricional formulada para atender as exigências de mantença e produção de ovos de acordo com as exigências das aves, de acordo com Silva et al. (2012) (Tab.1). As 80 codornas foram distribuídas em cinco tratamentos que consistiram na substituição do OS pelo OAS nos níveis de 2, 4, 6 e $8 \%$.

A quantidade de ração distribuída para cada ave foi de $50 \mathrm{~g}$ de ração/ave/dia durante cada período de 28 dias. As sobras de ração foram registradas a cada final de ciclo produtivo. As variáveis analisadas foram peso corporal ( $\mathrm{g}$ ) (sendo as aves pesadas individualmente no início do período experimental e ao final de cada ciclo), consumo de ração $(\mathrm{g})$, ganho de peso $(\mathrm{g})$, conversão alimentar (obtida a partir da relação entre o consumo total de ração e os quilos de ovos produzidos no período) e a produção diária de ovos por gaiola (para a obtenção do total de ovos produzidos por unidade experimental, foi realizado o somatório do número de ovos coletados de cada gaiola nos períodos avaliados).

A análise da composição do óleo ácido foi realizada pelo laboratório $C B O$ Análises Laboratoriais- Campinas, São Paulo (Tab. 2). Em virtude de não ter sido realizada a análise do OS utilizado neste estudo, foi feita uma revisão de literatura da composição em ácidos graxos maioritários presentes no OS (Tab. 3).

Os índices de acidez (IA) foram utilizados para avaliar a estabilidade lipídica, segundo normas da Association of Official Analytical Chemists (AOCS-1997). O IA foi avaliado por titulação das amostras com solução de hidróxido de sódio $(\mathrm{NaOH}) 0,01 \mathrm{~N}$ até o aparecimento de coloração rósea, indicando o ponto de viragem.

A qualidade do óleo foi avaliada seguindo a metodologia descrita pela AOCS (1997) utilizando-se dos coeficientes de extinção específica a $232 \mathrm{~nm}(K 232)$ e a $270 \mathrm{~nm}(K 270)$, onde em $232 \mathrm{~nm}$ são verificados a presença de compostos primários de oxidação, como peróxidos, hidroperóxidos e dienos conjugados; e em $270 \mathrm{~nm}$ são verificados a presença de compostos secundários de oxidação, como álcoois, cetonas e aldeídos. 
Tabela 1. Composição das dietas experimentais das codornas (Coturnix coturnix coturnix) alimentadas com níveis crescentes (\%) de óleo ácido de soja (OAS) em substituição ao óleo de soja (OS) na dieta

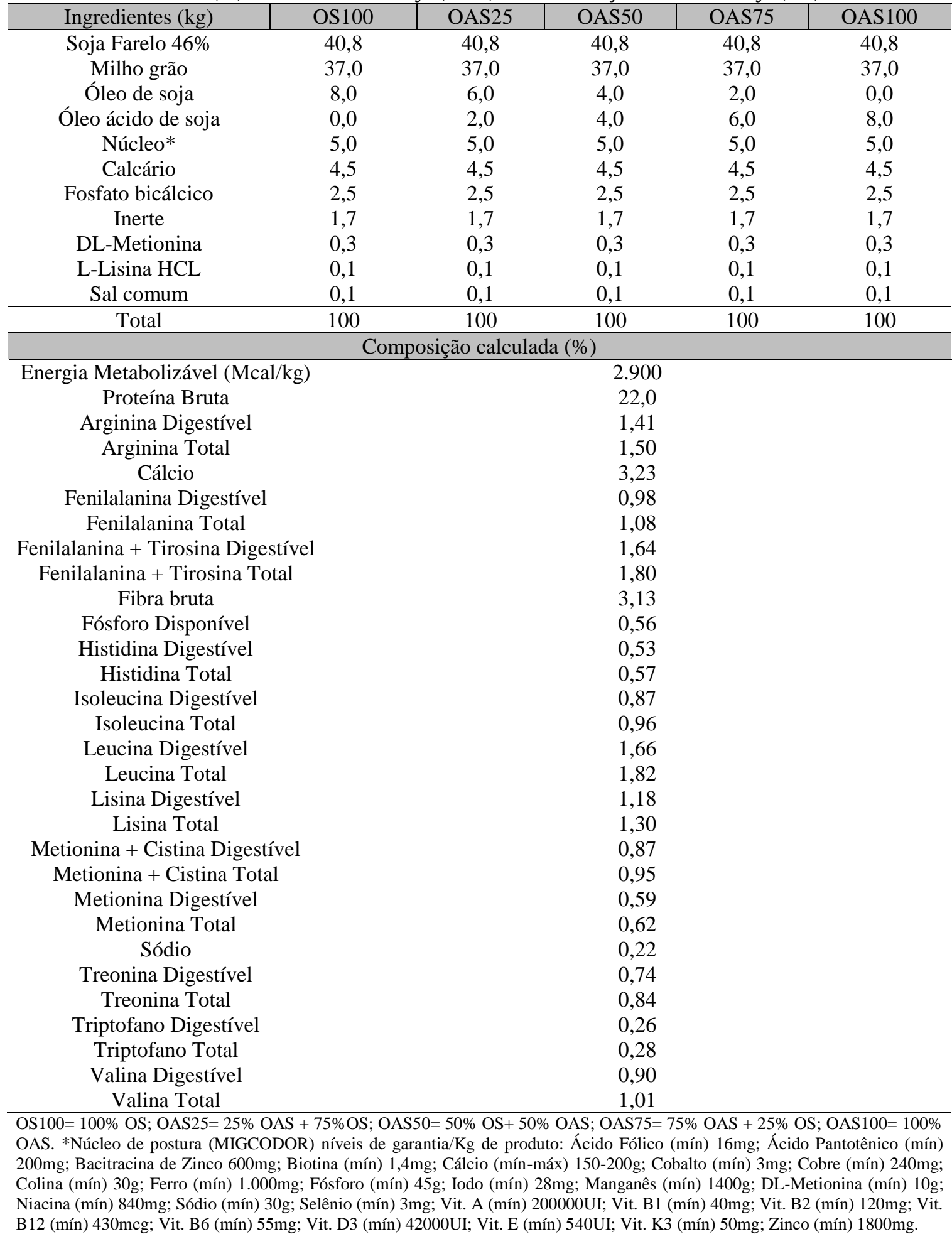


Tabela 2. Caracterização dos componentes do óleo ácido de soja (OAS)

\begin{tabular}{cc}
\hline Acidez em Ácido Oleico (\%, m/m) & 64,68 \\
Extrato Etéreo (\%) & 95,77 \\
\hline Composição em ácidos graxos (\%) \\
\hline Gorduras Insaturadas & 71,22 \\
Gordura Poli-insaturada & 46,36 \\
Gordura Monoinsaturada & 24,86 \\
Gorduras Saturadas & 24,55 \\
\hline Ácidos graxos maioritários (g/100g) & \\
\hline Ácido Linoleico (C18:2n6c) & 41,54 \\
Ácido Oleico (C18:1n9c) & 23,83 \\
Ácido Palmítico (C16:0) & 15,56 \\
Ácido Esteárico (C18:0) & 6,45 \\
\hline
\end{tabular}

Tabela 3. Ácidos graxos do óleo de soja refinado

\begin{tabular}{cccccc}
\hline Ácido Graxo & \multicolumn{5}{c}{ Óleo de soja refinado } \\
\cline { 2 - 6 }$(\mathrm{g} / 100 \mathrm{~g})$ & \multirow{2}{*}{ OAS } & Machado & Cunha & Aued-Pimentel & ANVISA \\
\hline Ácido Linoleico & 41,54 & 54,2 & 4608,48 & $48,11-54,65$ & $44,0-62,0$ \\
Ácido Oleico & 23,83 & 23,1 & 34,77 & $22,57-25,82$ & $19,0-30,0$ \\
Ácido Palmítico & 15,56 & 11,3 & 12,52 & $10,27-12,17$ & $7,0-14,0$ \\
Ácido Esteárico & 6,45 & 3,5 & 4,89 & $2,67-4,77$ & $1,4-5,5$ \\
\hline
\end{tabular}

Para o ensaio de metabolizabilidade das rações experimentais foi utilizado o marcador óxido férrico $\left(\mathrm{Fe}_{2} \mathrm{O}_{3}\right)$ que é um indicador do início e o término da coleta marcando as excretas que são realmente provenientes da ração que se deseja avaliar. O método de coleta total de excretas compreendeu um período de cinco dias de controle de consumo de ração e de coleta total de excretas em bolsas plásticas alocadas abaixo das gaiolas.

O marcador foi previamente preparado sendo adicionado na concentração de $3 \%$ na ração. As excretas foram coletadas diariamente as 9 horas da manhã, antes do arraçoamento, as mesmas após serem identificadas, foram pesadas $\mathrm{e}$ reservadas em bolsas plásticas sob refrigeração. Ao término do quinto dia de coleta, as amostras foram depositadas em bandejas de alumínio, pesadas (para obtenção do peso fresco), homogeneizadas e congeladas a $-20^{\circ} \mathrm{C}$ para posterior análise.

Para as análises das excretas as amostras foram descongeladas em temperatura ambiente por 24h. Após o descongelamento as amostras foram secas em estufa de circulação forçada de ar a $65^{\circ} \mathrm{C}$ durante 72 horas, sendo revolvidas diariamente para facilitar e garantir sua completa secagem. Posteriormente, as amostras de excretas e a ração experimental foram moídas em moinho tipo Willey, em peneira crivada de $1 \mathrm{~mm}$ de diâmetro, e submetidas à análise bromatológica.

As determinações de matéria seca e proteína bruta foram realizadas de acordo com a Association of Official Analytical Chemists (AOAC, 1995), onde foram avaliados os coeficientes de metabolizabilidade aparente e corrigida para nitrogênio $(\mathrm{N})$ da matéria seca, da proteína bruta e do extrato etéreo.

O cálculo do coeficiente de metabolizabilidade (CM) para a coleta total de excretas foi feito de acordo com a seguinte equação: CM $(\%)=$ Quantidade de nutriente da ração - Quantidade de nutriente da excreta / Quantidade de nutriente da ração x 100.

Os dados foram analisados de acordo com um delineamento experimental completamente casualizado sendo cada gaiola com duas aves considerada a unidade experimental. Foi realizada a análise de medidas repetidas usando o pacote "nlme - Linear and Nonlinear Mixed Effects Models" do pacote estatístico $\mathrm{R}$ de acordo com o seguinte modelo: $Y_{\mathrm{ijk}}=\mu+\mathrm{d}_{\mathrm{i}}+\mathrm{h}_{\mathrm{ij}}$ $+\mathrm{w}_{\mathrm{k}}+\mathrm{d}_{\mathrm{wik}}+\mathrm{e}_{\mathrm{ijk}}$, em que: $\mu=$ média geral; $\mathrm{d}_{\mathrm{i}}=$ efeito fixo da dieta (i= 1 a 5 dietas); $\mathrm{h}_{\mathrm{ij}}=$ efeito 
aleatório da gaiola dentro da dieta (tratamento; $\mathrm{j}=1$ a 8 , número de gaiolas por tratamento); $\mathrm{w}_{\mathrm{k}}$ = efeito fixo do período de postura $(\mathrm{k}=1$ a 2,); $\mathrm{d}_{\mathrm{wjk}}=$ interação entre dieta e período de postura (tratamento $\times$ período); $\mathrm{e}_{\mathrm{ijk}}=$ erro aleatório (erro residual).

A interação tratamento (dieta) $\times$ (período) foi considerado efeito fixo. Após realizar ANOVA foi utilizado o procedimento "LSM-least squares means" sendo as medias comparadas através do teste Tukey com um nível de significância $\mathrm{P}<0,05$.

Para estimar a associação existente entre os níveis de substituição do óleo de soja pelo óleo ácido de soja sobre as variáveis estudadas foi utilizada análise de regressão polinomial.

\section{RESULTADOS E DISCUSSÃO}

Na Tab. 4 são apresentados os resultados da análises de índice de acidez e os coeficientes de extinção específica (K232 e K270) das dietas contendo óleo ácido de soja. Foi observado um aumento linear do índice de acidez, K232 e $\mathrm{K} 270$ ( $\mathrm{P}<0,001)$, com o incremento de OAS na dieta das aves.

A variável K232 é principalmente um indicativo da conjugação de dienos e também da presença de compostos carbonilicos (Clodoveo et al., 2007). Enquanto que K270 é um dos marcadores usado para avaliar a formação de compostos secundários da oxidação (Gharby et al., 2015).

Tabela 4. Índice de acidez (IA) e coeficientes de extinção específica (K232 e K270) das dietas das codornas (Coturnix coturnix coturnix) alimentadas com óleo ácido de soja (OAS) em substituição ao óleo de soja (OS) na dieta

\begin{tabular}{|c|c|c|c|c|c|c|c|c|c|c|c|}
\hline \multirow[b]{2}{*}{ Item } & \multicolumn{5}{|c|}{ Dietas } & \multirow[b]{2}{*}{ EPM } & \multicolumn{5}{|c|}{ Probabilidade } \\
\hline & $\begin{array}{c}\text { OS } \\
100\end{array}$ & $\begin{array}{c}\text { OAS } \\
25 \\
\end{array}$ & $\begin{array}{c}\text { OAS } \\
50\end{array}$ & $\begin{array}{c}\text { OAS } \\
75 \\
\end{array}$ & $\begin{array}{c}\text { OAS } \\
100 \\
\end{array}$ & & Dietas & Dias & $\begin{array}{l}\text { Dietas } \\
\text { vs dias }\end{array}$ & Linear & Quadrática \\
\hline IA & 0,88 & 2,62 & 4,38 & 6,17 & 7,71 & 0,55 & $<, 0001$ & 0,16 & 0,02 & $<, 0001$ & $<, 0001$ \\
\hline K232 & 5,66 & 7,93 & 9,96 & 10,9 & 11,5 & 0,50 & $<, 0001$ & 0,83 & 0,08 & $<, 0001$ & $<, 0001$ \\
\hline K270 & 2,45 & 3,18 & 3,78 & 4,54 & 4,64 & 0,20 & $<, 0001$ & $<, 0001$ & $<, 0001$ & $<, 0001$ & $<, 0001$ \\
\hline
\end{tabular}

OS $=100 \%$ óleo de soja; OAS $25=25 \%$ OAS $+75 \%$ OS; OAS50 $=50 \%$ OS $+50 \%$ OAS; OAS75= 75\% OAS + 25\% $\mathrm{OS} ; \mathrm{OAS}=100 \%$ óleo ácido de soja

IA = índice de acidez

$\mathrm{EPM}=$ erro padrão da média.

Ambos k232 e k270 permitem avaliar a evolução oxidativa com base na relação A272nm/A232nm: quanto maior o valor da absorvência a $232 \mathrm{~nm}$, mais elevado será o conteúdo em peróxidos, correspondendo, portanto, ao início do processo de oxidação; pelo contrário, quanto maior for o valor de absorvência a $272 \mathrm{~nm}$, maior será o teor de produtos secundários presentes (Silva et al., 1999).

De acordo com Raber et al. (2009) a baixa qualidade do OAS comparada com o seu óleo de procedência é devida a elevada concentração de AGL e menor proporção da gordura total na forma de triglicerídios, que por sua vez proporcionam um maior grau de acidez ao OAS. Segundo Leeson e Summers (2001) os radicais livres originados durante o processo oxidativo se propagam destruindo os ácidos graxos essenciais, as proteínas, as vitaminas lipossolúveis e os carotenoides dos alimentos.

No presente estudo os maiores índices de acidez das dietas com níveis crescentes de OAS não provocaram mudanças nas variáveis produtivas $(\mathrm{P}>0,05)$, que foram semelhantes aos do tratamento controle. Os resultados médios de consumo diário de ração, porcentagem de produção, peso médio do ovo e a conversão alimentar por quilo de ovos são apresentados na Tab. 5 . 
Tabela 5. Desempenho produtivo de codornas (Coturnix coturnix coturnix) alimentadas com níveis crescentes (\%) de óleo ácido de soja (OAS) em substituição ao óleo de soja (OS) na dieta

\begin{tabular}{|c|c|c|c|c|c|c|c|c|c|c|c|}
\hline \multirow[b]{2}{*}{ Item } & \multicolumn{5}{|c|}{ Dietas } & \multirow[b]{2}{*}{ EPM } & \multicolumn{5}{|c|}{ Probabilidade } \\
\hline & $\begin{array}{c}\text { OS } \\
100\end{array}$ & $\begin{array}{c}\text { OAS } \\
25\end{array}$ & $\begin{array}{c}\text { OAS } \\
50\end{array}$ & $\begin{array}{c}\text { OAS } \\
75\end{array}$ & $\begin{array}{c}\text { OAS } \\
100\end{array}$ & & Dieta & Período & $\begin{array}{c}\text { Dieta } \\
\text { vs } \\
\text { período }\end{array}$ & Linear & Quadrática \\
\hline PESO (g) & 379 & 376 & 383 & 385 & 392 & 2,75 & 0,67 & 0,01 & 0,34 & 0,06 & 0,13 \\
\hline $\mathrm{CMD}$ (g/ave) & 44,0 & 44,1 & 43,9 & 43,9 & 43,8 & 0,06 & 0,53 & $<, 0001$ & 0,52 & 0,13 & 0,32 \\
\hline GP (g) & 4,20 & 0,40 & $-1,6$ & 5,00 & 2,30 & 1,90 & 0,82 & 0,007 & 0,30 & 0,95 & 0,79 \\
\hline PROD (\%) & 85,5 & 87,8 & 87,1 & 84,1 & 81,3 & 1,32 & 0,70 & 0,0003 & 0,07 & 0,19 & 0,23 \\
\hline PMO (g) & 12,9 & 13,6 & 14,0 & 13,3 & 13,5 & 0,19 & 0,72 & 0,007 & 0,87 & 0,55 & 0,42 \\
\hline $\mathrm{CA}(\mathrm{kg} / \mathrm{kg})$ & 3,51 & 3,26 & 3,27 & 3,35 & 3,60 & 0,07 & 0,62 & 0,0004 & 0,04 & 0,58 & 0,20 \\
\hline
\end{tabular}

OS $=100 \%$ óleo de soja; OAS25 $=25 \%$ OAS $+75 \%$ OS; OAS50 $=50 \%$ OS $+50 \%$ OAS; OAS75= 75\% OAS + 25\% OS; OAS $=100 \%$ óleo ácido de soja

$\mathrm{CMD}=$ consumo médio diário; $\mathrm{GP}=$ ganho de peso; $\mathrm{PROD}=$ produção de ovos; $\mathrm{PMO}=$ peso médio do ovo; $\mathrm{CA}=$ conversão alimentar

$\mathrm{EPM}=$ erro padrão da média

A análise de variância e o teste $\mathrm{F}$ para $\mathrm{o}$ ajustamento das equações de regressão mostraram não haver nenhum efeito significativo da utilização do OAS em níveis crescentes em até $8 \%$ em substituição ao óleo de soja na dieta das codornas sobre as variáveis de desempenho.

A falta de diferenças significativas nas variáveis produtivas $(\mathrm{P}>0,05)$ entre as dietas com níveis crescentes de OAS (AGL), é positiva no sentido de justificar o uso deste subproduto em codornas de postura.

Cabel et al. (1988) registraram que a degradação dos ingredientes e a formação de radicais livres em casos mais severos podem provocar sintomas de doenças como encefalomalácia, diástase exsudativa, distrofia muscular, necrose dos tecidos em frangos.

Neste sentido os níveis similares de produção alcançados são um indicativo de que os mecanismos antioxidantes foram capazes de manter a homeostase e saúde nas aves, apesar de que a composição de produtos da peroxidação de lipídios da dieta aumentava conforme os níveis de inclusão de óleo ácido de soja nas dietas.

A análise realizada no OAS utilizado na presente pesquisa demonstrou que a acidez do produto era de apenas $64,68 \%$, ou seja, $35,32 \%$ de seu total poderia ser composto por misturas de mono, di e/ou triglicerídeos (Tab. 2). Estes resultados concordam com Vieira et al. (2002) que ressaltaram que embora o OAS seja normalmente considerado composto apenas por AGL, os autores encontraram uma proporção de até $30 \%$ do total do óleo que não estava na forma livre (AGL).

Os resultados produtivos mesmo com a inclusão de óleo ácido foram satisfatórios em comparação com outros trabalhos. No presente estudo foi encontrada uma média de produção de ovos/período de $85 \%$ que é considerado um bom resultado tratando-se de codornas com 30 semanas de idade que foram selecionadas por peso corporal durante por 17 gerações (peso médio $=380 \mathrm{~g}$ ).

Eichener et al. (2004) não encontram diferenças no ganho de peso, consumo de ração e conversão alimentar das aves alimentadas com OAS. Vila e Esteve-Garcia, (1996) verificaram que o crescimento de frangos não foi afetado pelo consumo de rações compostas por lipídios com alto conteúdo de AGL. Pardío et al. (2001) não constataram perdas de desempenho e relataram ganho de peso em frangos de corte com a inclusão de OAS na dieta por um período de sete semanas.

Bornstein e Lipstein (1963) encontraram redução de no máximo $9 \%$ de absorção do OAS referente ao OS, indicando que $\mathrm{o}$ valor energético total das duas fontes é bastante próximo. Mais recentemente, Roll et al. (2014) não verificaram diferenças no consumo, ganho 
de peso e conversão alimentar em frangos de corte alimentados com óleo ácido de palma $(\mathrm{AGL}=88,6 \%)$, embora a digestibilidade dos ácidos graxos saturados tenha sido menor em comparação com uma dieta com óleo de palma nativo $(\mathrm{TG}=79,7 \%)$.

Porém estudos indicam prejuízos com a utilização de óleos ácidos na nutrição de aves, como exemplo, Gaiotto et al. (2000) que substituindo o OS em proporções iguais pelo OAS, sem fazer correções para os níveis de energia das dietas, constataram perdas no desempenho das aves comparadas com as que receberam dietas com o óleo de soja.

Vieira et al. (2002) em um experimento com frangos de corte avaliando dietas que continham OAS e óleo degomado de soja (ODS), observaram um melhor aproveitamento da energia pelas aves que foram alimentadas com as dietas que continham ODS.

Da mesma forma, Lara et al. (2005) encontraram um menor ganho de peso e consumo de ração em frangos alimentados com OAS em comparação aos que receberam óleo de soja na dieta, embora a conversão alimentar não tenha sido afetada. Sklan (1979) também observou uma redução na capacidade de absorção dos frangos que foram alimentados com óleo ácido, relatando que essas aves apresentaram menor proporção de micelas no intestino delgado.

No presente estudo, foram encontradas médias de consumo em torno de $44 \mathrm{~g} / \mathrm{dia}$, representando um consumo de $8 \mathrm{~g} /$ dia a mais com relação aos dados de Oliveira (2003) que relatam que a codorna da linhagem europeia consome em torno de $36 \mathrm{~g} /$ dia de ração na fase de produção. Cabe ressaltar que a codornas utilizadas para este experimento representam a $17^{\mathrm{a}}$ geração de codornas de corte melhoradas geneticamente para o maior ganho de peso, pesando em média $380 \mathrm{~g}$.

$\mathrm{Na}$ Tab. 6 são apresentados os resultados referentes aos coeficientes de metabolizabilidade das cinzas (CMCZ), da gordura bruta (CMGB), da matéria seca (CMMS) e da proteína bruta (CMPB). Com exceção do CMGB, todos os demais coeficientes não apresentaram diferenças significativas.

Tabela 6. Coeficientes de metabolizabilidade em codornas fêmeas (Coturnix coturnix coturnix) alimentadas com níveis crescentes (\%) de óleo ácido de soja (OAS) em substituição ao óleo de soja (OS) na dieta

\begin{tabular}{|c|c|c|c|c|c|c|c|c|}
\hline \multirow[b]{2}{*}{ Item } & \multicolumn{5}{|c|}{ Dietas } & \multirow[b]{2}{*}{ EPM } & \multicolumn{2}{|c|}{ Probabilidade } \\
\hline & $\begin{array}{c}\text { OS } \\
100 \\
\end{array}$ & $\begin{array}{c}\text { OAS } \\
25 \\
\end{array}$ & $\begin{array}{c}\text { OAS } \\
50\end{array}$ & $\begin{array}{c}\text { OAS } \\
75 \\
\end{array}$ & $\begin{array}{c}\text { OAS } \\
100\end{array}$ & & Linear & Quadrática \\
\hline CMCZ & 30,16 & 28,26 & 24,69 & 28,17 & 31,39 & 1,57 & 0,83 & 0,43 \\
\hline CMGB & 93,89 & 91,74 & 88,85 & 89,94 & 93,48 & 0,71 & 0,61 & 0,02 \\
\hline CMMS & 72,48 & 70,57 & 69,04 & 69,37 & 73,55 & 0,71 & 0,85 & 0,054 \\
\hline CMPB & 47,67 & 42,96 & 28,41 & 36,23 & 54,58 & 3,55 & 0,90 & 0,08 \\
\hline
\end{tabular}

OS $100=100 \%$ OS $;$ OAS $25=25 \%$ OAS $+75 \%$ OS; OAS $50=50 \%$ OS $+50 \%$ OAS; OAS75 $=75 \%$ OAS $+25 \%$ OS; OAS100 $=100 \%$ OAS

$\mathrm{CMCZ}=$ coeficiente de metabolizabilidade de cinzas; $\mathrm{CMGB}=$ coeficiente de metabolizabilidade da gordura bruta; $\mathrm{CMMS}=$ coeficiente de metabolizabilidade da matéria seca; $\mathrm{CMPB}=$ coeficiente de metabolizabilidade da proteína bruta.

$\mathrm{EPM}=$ erro padrão da média.

A análise de regressão comprovou efeito quadrático dos níveis crescentes de inclusão de OAS em substituição ao OS na dietas das aves sobre o coeficiente de metabolizabilidade da gordura bruta $\left(\mathrm{CMGB}=94,31-1,186 x+0,018 \mathrm{x}^{2}\right.$, $\left.\mathrm{R}^{2}=0,28, \mathrm{P}=0,02\right)$.
O CMGB é reduzido até o nível de $50 \%$ de inclusão de OAS, sendo aumentado a partir deste nível. Com o aumento do nível de OAS na ração, houve redução significativa na digestibilidade da gordura, confirmando a importância do nível de acréscimo de OAS sobre esta variável. 
No presente estudo, observa-se (Tab. 6) que o CMGB foi de $93,48 \%$ para o OAS, ao passo que para a OS foi de $93,89 \%$, o que mostra uma semelhante eficiência das codornas de corte na utilização do OAS comparado com o OS.

O efeito quadrático na redução do CMGB do OAS denota uma semelhança de digestibilidade do óleo ácido em relação ao OS, e ao contrário do esperado indica que a mistura de óleo não favoreceu a digestibilidade da gordura, conforme é relatado na literatura.

Desse modo, Renner e Rill (1961), reportaram que os ácidos graxos saturados (AGS), palmítico (C16:0) e esteárico (C18:0), tiveram uma absorção de $30 \%$ e $20 \%$, respectivamente, quando utilizaram uma mistura contendo $50 \%$ de ácidos graxos insaturados (AGI), sendo que essa absorção viu-se aumentada para $51 \%$ (C16:0) e 36\% (C18:0) quando foi aumentando para $62 \%$ a quantidade de AGI da mistura. Os mesmos autores ao elevarem a proporção de AGI para $76 \%$, observaram uma absorção de $84 \%$ para o ácido graxo palmítico e $76 \%$ para o ácido graxo esteárico. Evidenciando a importância da maior proporção de ácidos graxos insaturados com relação aos saturados nas gorduras para um melhor aproveitamento das mesmas.

Em resumo, o produto comercial OAS utilizado neste estudo apresentou em sua composição 24,55\% dos lipídios na forma de AGS e 71,22\% dos lipídios na forma de AGI, sendo que destes 46,36\% eram de ácidos graxos poli-insaturados e $24,86 \%$ de ácidos graxos monoinsaturados. A elevada concentração de AGI do OAS pode ter facilitado a absorção dos lipídios da dieta de forma geral, melhorando assim a utilização da gordura das dietas pelas aves reduzindo a diferença no aproveitamento das gorduras entre as dietas testadas.

A relação de AGL com TG intactos é importante pois os AGL são absorvidos com menor eficiência do que aqueles provenientes de TG. Isso acontece em razão dos MG serem essenciais para a incorporação de ácidos graxos insolúveis no complexo micelar. Quando os AGL são fornecidos como única fonte de lipídios não existe MG suficientes e a absorção fica prejudicada (Blanch et al., 1995), evidenciando que os AGL necessitam de MG para serem absorvidos na forma de micelas, juntamente com sais biliares. Leeson e Summers (2001) registram que entre 50 a $78 \%$ dos TG da dieta são hidrolisados a 2-monoglicerídios e absorvidos nessa forma.

Cabe ressaltar que a maioria dos estudos encontrados na literatura foram realizados com frangos de corte. De acordo com Silva et al. (2012), as codornas apresentam aproveitamento e exigências diferente daquelas para frangos de corte e galinhas poedeiras, assim como o tempo de passagem do alimento pelo trato digestivo das codornas é mais acelerado, variando de 60 a 90 minutos. A taxa de passagem pelo aparelho digestório também está relacionada com outras variáveis como, por exemplo, a quantidade de alimento ingerido, a composição da dieta, o aspecto físico do alimento entre outros (Rezende et al., 2008).

Todos estes fatores podem ter influência sobre a ausência de diferenças significativas nas variáveis de desempenho e metabolizabilidade encontradas no presente estudo com a utilização de níveis crescentes de óleo ácido na dieta dessas aves. Somando-se a esses fatores, o curto período de suplementação (56 dias), aliado as características anatômicas das codornas em termos de tamanho e comprimento dos órgãos do trato gastrointestinal, de particularidades fisiológicas e às vezes, de hábitos alimentares podem explicar os resultados contraditórios da literatura em geral.

Por outro lado, as diferentes técnicas de processamento das gordura e óleos em diferentes regiões, podem gerar produtos com diferentes composições químicas. Isto poderia explicar os resultados divergentes encontrados na literatura em relação ao óleo ácido.

\section{CONCLUSÕES}

O desempenho das codornas de corte em postura não é afetado de forma negativa pelo acréscimo de OAS na dieta das aves e a digestibilidade da gordura apresenta-se com um ajuste quadrático, ocorrendo perda de eficiência com a mistura dos óleos ao nível de 50\%, indicando ausência de sinergismo entre as misturas de óleos. No nível de substituição de $100 \%$ do OS pelo OAS, os parâmetros avaliados 
mostram-se equivalentes aos do tratamento controle.

\section{REFERÊNCIAS}

AOAC - ASSOCIATION OF OFFICIAL ANALYTICAL CHEMISTS. Official methods of analysis of the Association of the Analytical Chemists. 16ed. Washington, 1995. 1094p.

AOAC - ASSOCIATION OF OFFICIAL ANALYTICAL CHEMISTS. Official methods of analysis of the Association of the Analytical Chemists. 17ed. Washington, 1997. 1094p.

BLANCH, A.; BARROETA, A.C.; BAUCELLS, M.D.; PUCHAL, F. The nutritive value of dietary fats in relation to their chemical composition: apparent fat availability and metabolizable energy in two-week-old chicks. Poult. Sci., v.74, p.1335-1340, 1995.

BORNSTEIN, S.; LIPSTEIN, B. Some unusual waste vegetable oils as fat supplements in practical broiler rations. World's Poult. Sci. J., London, v.19, p.172-184, 1963.

CABEL, M.C.; WALDROUP, W.; SHERMER, W.D. et al. Effects of ethoxyquin feed preservative and peroxide level on broiler performance. Poult. Sci., v.67, p.1725-1730, 1988.

ClODOVEO, M.L.; DELCURATOLO, D.; GOMES, T.; COLELLI, G. Effect of different temperatures and storage atmospheres on Coratina olive oil quality. Food Chem. v.102, p.571-576, 2007.

DE GROOTE, G. Energetic evaluation of unstabilized and stabilized fish meals in terms of metabolizable energy and net energy for maintenance and growth. Feedstuffs, v.40, p.26$27+54-61,1968$.

DIONELLO, N.J.L; CORREA, G.S.S; SILVA, M.A. et al. Estimativas da trajetória genética do crescimento de codornas de corte utilizando modelos de regressão aleatória. Arq. Bras. Med. Vet. Zootec. Belo Horizonte, v.60, p.454-460, 2008.

EICHNER, G.; VIEIRA, S.L.; VIOLA, E.S. Desempenho, rendimento de carcaça e umidade de cama de frangos de corte consumindo dietas formuladas com óleo ácido de soja. Rev. Bras. Ciênc. Avic., Campinas, p. 62, 2004.
FREITAS, E.R.; SAKOMURA, N.K.; NEME, R.; SANTOS, A.L. dos. Valor energético do óleo ácido de soja para aves. $P A B$, v.40, p.241246, 2005.

GAIOTTO, J.B.; MENTEN, J.F.M.; RACANICCI, A.M.C.; LAFIGLIOLA, M.C. Óleo de soja, óleo ácido de soja e sebo bovino como fontes de gordura em rações para frangos de corte. Rev. Bras. Ciênc. Avic., Campinas, v.2, p.219-227, 2000.

GHARBY, S.; HARHAR, H.; BOUZOUBAÂ, Z. et al. The Effect of Storage Conditions and Roasting Kernels on Extra Virgin Argan Oil Quality. J. Mater. Environ. Sci., v.6, p.254-263, 2015.

LARA, L.J.; BAIÃO, N.C.; CANÇADO, S.V. et al. Efeito de fontes lipídicas sobre $\mathrm{o}$ desempenho de frangos de corte. Arq. Bras. Med. Vet. Zootec., v.57, p.792-798, 2005.

LEESON, S; SUMMERS, J.D. Nutrition of the chicken. Ontario, UNIVERSITY BOOKS, 4ed., 2001. 413p.

LEWIS, D.; PAYNE, C.G. Fats and amino acids in broiler rations. 3. Supplementation with different levels and types of fat. Br. Poultry Sci., v.4, p.13-18, 1963.

MURAKAMI, A.E.; FURLAN, A.C. Pesquisas na nutrição e alimentação de codornas em postura no Brasil. In: SIMPÓSIO INTERNACIONAL DE COTURNICULTURA, 1, 2002, Lavras, MG. Anais... Lavras: Universidade Federal de Lavras, 2002. p.113120.

PARDÍO, V.T.; LANDIN, L.A.; WALISZEWSKI, K.N. et al. The effect of acidified soapstocks on feed conversion and broiler skin pigmentation. Poult. Sci., v.80, p.1236-1239, 2001.

RABER, M.R.; RIBEIRO, A.M.L.; KESSELER, A.M.; ARNAIZ, V. Suplementação de glicerol ou de lecitina em diferentes níveis de ácidos graxos livres em dietas para frangos de corte. Ciênc. Anim. Bras., v.10, p. 745-753, 2009.

RENNER, R.; HILL, F.W. Factors affecting the absorbability of saturated fatty acids in the chick. J. Nutr., v.74, p.254-258, 1961. 
REZENDE, M.J.; FLAUZINA, L.P.; MCMANUS, C.; DE OLIVEIRA, L.Q.M. Desempenho produtivo e biometria das vísceras de codornas francesas alimentadas com diferentes níveis de energia metabolizável e proteína bruta. Acta Sci. Anim. Sci., v.26, p.353-358, 2008.

ROLL, A.P.; VILARRASA, E.; BARROETA, A.C. Proporções de mono e digliceridos e ácidos graxos livres de óleo de palma na dieta de frangos de corte. In: XIII SEMINÁRIO TÉCNICO CIENTÍFICO DE AVES E SUÍNOS

AveSui 2014. Florianópolis: Anais... Florianópolis [s.n.] 2014. (Resumo)

SAKAMOTO, M.I.; MURAKAMI, A.E.; SOUZA, L.M.G. et al. Valor energético de alguns alimentos alternativos para codornas japonesas. R. Bras. Zootec., v.35, p.818-821, 2006.

SILVA, F.A.; BORGES, M.F.M.; FERREIRA, M.A. Métodos para avaliação do grau de oxidação lipídica e da capacidade antioxidante. Quím. Nova, v.22, p.94-103, 1999.

SILVA, J.H.V.; JORDÃO FILHO, J.; COSTA, F.G.P. et al. Exigências nutricionais de codornas. Rev. Bras. Saúde Prod. Ani., Salvador, v.13, p. 775-790, 2012.
SKLAN, D. Digestion and absorption of lipids in chicks fed triglycerides or free fatty acids: synthesis of monoglycerides in the intestine. Poult. Sci., v.58, p.885-889, 1979.

TREAT, C.M.; REID, B.L.; DAVIES, R.E.; COUCH, J.R. Effect of animal fat and mixtures of animal and vegetable fats containing varying amounts of free fatty acids on performance of cage layers. Poult. Sci., v.39, p.1550-1555, 1960.

VIEIRA, S.L.; RIBEIRO, A.M.L.; KESSLER, A.M. Utilização da energia de dietas de frangos de corte formuladas com óleo ácido de soja. Rev. Bras. Ciênc. Avíc., Campinas, v.4, p.127139, 2002.

WALDROUP, P.W.; WATKINS, S.E.; SALEH, E.A. Comparison of two blended animalvegetable fats having low or high free fatty acid content. J. Appl. Poultry Res., v.4, p.41-48, 1995. 